\title{
Out of Sight, Out of Sync: Understanding Conflict in Distributed Teams
}

\author{
Pamela J. Hinds • Diane E. Bailey \\ Center for Work, Technology and Organization, Department of Management Science and Engineering, \\ Stanford University, Stanford, California 94305-4026 \\ phinds@stanford.edu・debailey@stanford.edu
}

\begin{abstract}
The bulk of our understanding of teams is based on traditional teams in which all members are collocated and communicate face to face. However, geographically distributed teams, whose members are not collocated and must often communicate via technology, are growing in prevalence. Studies from the field are beginning to suggest that geographically distributed teams operate differently and experience different outcomes than traditional teams. For example, empirical studies suggest that distributed teams experience high levels of conflict. These empirical studies offer rich and valuable descriptions of this conflict, but they do not systematically identify the mechanisms by which conflict is engendered in distributed teams. In this paper, we develop a theory-based explanation of how geographical distribution provokes team-level conflict. We do so by considering the two characteristics that distinguish distributed teams from traditional ones: Namely, we examine how being distant from one's team members and relying on technology to mediate communication and collaborative work impacts team members. Our analysis identifies antecedents to conflict that are unique to distributed teams. We predict that conflict of all types (task, affective, and process) will be detrimental to the performance of distributed teams, a result that is contrary to much research on traditional teams. We also investigate conflict as a dynamic process to determine how teams might mitigate these negative impacts over time.

(Distributed Work; Distributed Teams; Virtual Teams; Conflict)
\end{abstract}

In response to a variety of factors that characterize the modern economy-including the global expansion of the marketplace and the businesses that serve it, the rise in mergers and acquisitions, and heightened competitive pressures to reduce the time to develop productsorganizations increasingly are assembling teams whose members are drawn from sites far and near. Geographically distributed teams face a number of unique challenges, including being coached from a distance, coping with the cost and stress of frequent travel, and dealing with repeated delays (Armstrong and Cole 2002). Many scholars and practitioners have noted and expressed concern about one such challenge facing these teams: the prevalence and severity of conflict. Justifying their concern, reports from the field indicate that conflict is disruptive to performance in distributed teams.

Field studies further indicate that geographically distributed teams may experience conflict as a result of two factors: The distance that separates team members and their reliance on technology to communicate and work with one another. Distance and technology mediation have gone unexplored in existing models of conflict and performance in teams because their authors, for the most part, assumed that team members were collocated and communicating face to face. As a result, whether these two factors spur new antecedents of conflict is not known, nor is it clear how conflict in distributed teams might be reduced. In this paper, we consider the possibility that distance and technology mediation give rise to conflict in distributed teams. We also examine how conflict might manifest itself over time as members of distributed teams learn how to work and communicate across distances and use technology more effectively.

Geographically distributed teams, whose members reside in different cities, countries, or continents, share a number of properties commonly associated with traditionally conceived teams. Namely, they are groups of individuals that work together interdependently to accomplish a task, constitute distinct social entities, and jointly manage their team boundaries (Cohen and Bailey 1997, Hackman 1987).

Recent studies demonstrate the kinds of problems that arise uniquely in the case of distributed teams and that render questionable the comprehensiveness of past models of group conflict and performance. For example, Armstrong and Cole (2002) reported that conflicts in geographically distributed teams went unidentified and unaddressed longer than conflicts in collocated teams. Beyond such empirical evidence, however, there is no comprehensive theory-driven prediction and explanation 
for conflict in distributed teams. It is not known whether conflict in distributed teams is triggered in a manner similar to that for traditional teams, nor is it clear whether the impact of conflict on performance is the same as in traditional teams. We investigate these issues by reviewing findings from research on distance and technology mediation and blending those findings with research on conflict in traditional teams. We also consider evidence from the growing number of empirical studies of distributed teams, which provide support for the propositions that we build inductively. Our theoretical analysis is intended to help establish a roadmap for future empirical work on distributed teams.

Our analysis reveals that geographical distribution will have a significant impact on each type of group conflict proposed in recent organizational studies: task, affective, and process. Task conflict refers to disagreements focused on work content. Affective conflict (sometimes referred to as relationship or emotional conflict) refers to team disagreements that are characterized by anger or hostility among group members. Process conflict refers to disagreements over the team's approach to the task, its methods, and its group processes. Affective conflict has been differentiated from task conflict (Eisenhardt et al. 1997, Pelled 1996, Pelled and Adler 1994) and from process conflict (Jehn 1997) partly in an effort to explain contradictory findings regarding the impact of conflict on team performance. Our analysis not only identifies a number of new antecedents for each type of conflict in distributed teams, it also reveals that the impact of group conflict will in some cases be different for distributed teams than for traditional, collocated teams. For example, task conflict has been found to be beneficial for performance on many traditional teams, but we contend that it will not be so for their distributed counterparts.

Although we predict worse outcomes for distributed teams, we acknowledge that they have certain advantages over collocated teams. Distributed teams enable firms to take advantage of expertise around the globe, to continue work around the clock, and to create closer relationships with far-flung customers. We argue that these benefits will be diminished by the conflict engendered by distance and technology mediation, but we acknowledge that distributed teams may, at times, be the only viable option for achieving organizational goals. We therefore extend our analysis to consider conflict as a dynamic rather than a static process to illustrate how teams might mitigate the negative effects of distribution over time. Although we contend that the negative effects cannot be fully overcome, the preventative measures we identify may facilitate the performance of distributed teams when the realities of business dictate their use.
In our analysis, we specify that distributed teams differ from traditional teams in only two respects: members separated by distance and forced to rely on technologies to mediate their communication and collaborative work. At first glance, this approach may appear to rule out other traits that might distinguish a distributed team from a collocated one. We contend that all other traits that may be associated with geographical distribution derive from distance or technology mediation, and we consider them in our analysis of these two factors. For example, some, but not all, distributed teams may experience incongruent temporal rhythms because members work in different time zones, but different time zones occur as a result of distance. Likewise, distributed teams may have members from different cultures. Beyond the impact of distance, distributed teams are no more or less likely than collocated teams to have members working at different times or to have a culturally diverse membership. Moreover, the effects of technology mediation are distinct from those of distance. Although distributed team members must rely on technologies because of their distance, technology mediation has impacts even for teams that are collocated, and that at times choose to rely heavily on technology rather than meet face to face (Mortensen and Hinds 2001). Among the many traits that might distinguish geographically distributed teams from collocated ones, separation by distance and heavy reliance on mediating technologies are the only two factors that hold true for all distributed teams.

In the balance of the paper, we use the term "group" to refer to ad hoc collections of individuals brought together solely for the purpose of research, typically for a short period, and with limited shared past or anticipated future (e.g., as in experimental studies). Although groups of this nature may not closely resemble organizational teams, studies employing them have closely examined the effects of technology mediation and have strong, consistent findings that are extremely relevant to the case of distributed teams. We reserve the term "team" for groups in organizations. We often abbreviate "geographically distributed teams" to simply "distributed teams," but in doing so do not intend to include other forms of distributed teams (e.g., organizationally distributed teams) whose experiences with conflict we do not consider in this paper. Finally, we treat as synonymous the terms "traditional teams" and "collocated teams" even though what is new about distributed teams is their increasing prevalence, not their existence. Collocated teams represent how scholars have traditionally conceived of teams. 


\section{Distance and Conflict}

In this section, we build on research demonstrating that distance has a detrimental impact on team members' shared context, familiarity, and friendship, all factors that can heighten conflict in teams. We further argue that distance is likely to bring with it increased heterogeneity, particularly cultural differences, that will reduce the similarity of team members. We describe how the negative impact of distance on shared context, familiarity, friendship, and homogeneity will precipitate conflict for distributed teams.

\section{Shared Context}

Because they are distant from each other, members of distributed teams may have difficulty establishing a shared context. Different contexts may derive from and be revealed in different work and geographic environments, different technologies, and different cultures. Occupying different physical contexts makes it more difficult to make and interpret references to objects of interest (Schober 1998) and to co-orient in a particular context. For example, in a study of the use of new machines in a factory, Tyre and von Hippel (1997) observed that engineers and operators had trouble resolving equipment problems over the phone because the engineers needed to "see for themselves" the technology in context. In this way, distance fosters different perspectives on and information about the work in which distributed team members are engaged. In the absence of a shared context, team members will have difficulty developing mutual understanding (Fussell and Krauss 1992, Clark and Brennan 1991). When team members have different understandings of the task, task conflict is likely to result (Jehn et al. 1997). Moreover, when team members' understanding of the issues differs, conflict is difficult to resolve (Brehmer 1976).

Team members who lack a sense of a shared context as a result of distance also are likely to adhere to different norms. Offices, meetings rooms, cafeterias, and so forth are associated with behavioral norms and mental schemas that affect team members' behaviors and expectations of one another (Kiesler and Cummings 2002). Team members who do not share the same social setting may have different perceptions about what behaviors are appropriate, consequently holding one another to different standards. Karnoe (1995), for example, observed that Danish and American workers used different paradigms for understanding problems and solutions. He attributed these differences to disparities in local routines and behavioral norms. Armstrong and Cole (2002) also observed that site-specific cultures and expectations acted as significant sources of misunderstandings and conflict between distant sites. In short, dissimilar paradigms, norms, and behavioral expectations are likely to result in more task and affective conflict.

In addition to disrupting shared understanding and the development of common behavioral norms, occupying different contexts may make it difficult for groups to establish a shared temporal rhythm, or to become "entrained." Ancona and Chong (1996) argued that groups establish a rhythm that serves as a powerful coordination mechanism. Isomorphic processes at the group level may create similarity in temporal cycles as team members signal to each other the pace and timing of activities (Ancona and Chong 1996). In distributed teams, signals among distant team members may be difficult to observe and interpret. Grinter et al. (1999) found that members of distributed software development teams, regardless of the way they structured their work, were "constantly surprised" and confused about the activities of their distant colleagues. In the absence of triggers available to traditional teams, distributed teams have been observed to use face-to-face meetings and other interactions to establish the rhythm of the team (Maznevski and Chudoba 2000). Such difficulties in developing a shared temporal rhythm may make coordination in distant teams more fraught with conflict as team members continually find their expectations of others unmet and their work processes "out of sync." We posit that distant team members will experience more incongruent temporal rhythms, which in turn will engender unfavorable attributions (Cramton 2002) and process conflict as confusion arises about who is doing (or has done) what and when.

\section{Familiarity}

Whereas shared context either exists or does not, familiarity can build over time when people are continually copresent. Mere exposure to others powerfully affects peoples' feelings about one another (Zajonc 1968). As compared to collocated teams, distributed team members tend to receive less passive information about their distant colleagues. Armstrong and Cole (2002) reported that distance blocked casual visual observation, consequently inhibiting learning across sites. Similarly, proximity increases the amount of informal interaction that can occur among team members. When people are collocated, the number of casual encounters, unplanned conversations, and multipurpose interactions increases (Kraut et al. 2002). Such opportunities promote familiarity as team members learn about the personalities, concerns, and work processes of others. Familiarity, in turn, is associated with reduced conflict. Deutsch (1969) reported that lack of familiarity increased conflict 
about roles and responsibilities. Similarly, Goodman and Leyden (1991) found that not being familiar with the work habits of other team members increased coordination problems in the team. These studies suggest that process conflict will be greater in distributed teams because their members have fewer opportunities to become familiar with one another.

\section{Friendship}

Proximity also is associated with friendship. Festinger et al. (1950) found that graduate students and their families who were randomly assigned to housing near one another were more likely to become friends. Friendship is easier to establish when people casually encounter one another and interact spontaneously. Grinter et al. (1999) observed that distant team members had difficulty building rapport and developing long-term relationships without meeting face to face. Surprisingly, when team members are friends, conflict, particularly affective conflict, is likely to be more prevalent. In an experiment conducted with business students, Shah and Jehn (1993) found that friend groups experienced more emotional conflict than groups of strangers working on decision-making tasks. Murnighan and Conlon (1991) also reported higher levels of conflict in string quartets in which members were friends, but friend groups were better able to manage conflict successfully. Because bonds of friendship are built on trust, expressing affective conflict may be perceived as safer and more readily accepted. These studies suggest that friendship increases affective conflict in teams, but that these teams also are better able to harness the conflict to improve task performance. In sum, research on friendship suggests that distributed teams will experience less friendship and, thus, less affective conflict.

\section{Homogeneity}

Finally, distance is likely to reduce homogeneity among team members by increasing demographic heterogeneity, particularly ethnic or racial heterogeneity. In a comparison of collocated and distributed product development teams, Mortensen and Hinds (2001) reported that distributed teams were somewhat more culturally diverse than collocated teams. Such diversity has been shown to increase task and affective conflict (O'Reilly et al. 1997, Pelled 1996) because diversity prompts different perspectives on, and approaches to, work and fuels different attitudes, beliefs, and expectations. In their review, Williams and O'Reilly (1998, p. 115) conclude that over 40 years of research has found that "diverse groups are more likely to be less integrated, have less communication, and have more conflict." We thus expect distant teams to be more heterogeneous and to experience more task and affective conflict.

\section{Overall Impact of Distance}

In sum, the social and psychological effects of distance are likely to lead to more task and process conflict due to challenges resulting from different perspectives, inconsistent norms, incongruent temporal rhythms, reduced familiarity, and demographic heterogeneity. The effect of distance on affective conflict, however, is less immediately apparent. On one hand, distance should lead to more affective conflict as team members adhere to inconsistent norms and attempt to work through demographic differences. On the other hand, distance may lessen affective conflict because team members do not have a basis of friendship that would enable them to express affective conflict openly.

Although one might expect these opposing forces to result in little ultimate impact on affective conflict, we argue that distance will heighten affective conflict. Our reasoning lies in the relationship between task and affective conflict. Task conflict can lead to increased affective conflict, especially in teams with low trust, perhaps because low trust leads to more faulty attributions regarding the source of the disagreement (Simons and Peterson 2000). When trust is missing, team members are more likely to question others' intentions and make attributions that do not adequately account for situational factors. In distributed teams, trust can be fragile and often fractures rapidly (Jarvenpaa and Leidner 1999). Thus, although distance may not directly lead to affective conflict, increased task conflict will result in more affective conflict for distributed teams. This argument is consistent with Cramton's (2001) observations that reduced information about team members' actions on distributed teams will lead to more harsh attributions about their intentions. Overall, we propose that distance will engender conflict of all types for distributed teams.

Proposition 1. As a result of the different perspectives and norms and reduced homogeneity that it occasions, distance engenders task conflict in teams.

Proposition 2. As a result of the different norms and temporal rhythms and reduced homogeneity that it occasions, distance engenders affective conflict in teams, despite the ameliorating effect of reduced friendship.

Proposition 3. As a result of the different temporal rhythms and reduced familiarity that it occasions, distance engenders process conflict in teams.

Proposition 4. As a result of the reduced trust that it occasions, distance engenders a strong positive relationship between task conflict and affective conflict. 


\section{Technology Mediation and Conflict}

When distributed team members wish to communicate with one another or work together, they typically employ technology. With limited opportunities for talking face to face, they may hold discussions by phone or e-mail. Unable to carry a document to a colleague's cubicle, they may resort to electronic options, such as posting it on a Web page or storing it in an intranet-accessible directory. In this manner, technology mediates both communication and collaborative work for distributed teams. Our reading of prior research suggests that the effects of technology mediation can be categorized according to their impact on relational outcomes, information transfer, and coordination. Although significant interest has been paid to the relational outcomes of technology mediation for distributed teams, we conclude that issues of information transfer and coordination may have an equal, if not greater, bearing on group conflict.

The bulk of prior research on technology mediation examines use of communication technologies such as computer-supported meeting systems, audio- and videoconferencing, and e-mail. Unfortunately, relatively little work considers the use of information technologies such as shared electronic workspaces or version control software that mediate collaborative work absent direct interpersonal communication. Such technologies are equally important for a distributed team's functioning because team members spend only a portion of their time meeting or otherwise communicating with one another. A few technologies support both communication and information sharing and have been studied (see Mark et al. 1999, Olson and Teasley 1996, Orlikowski 1992). As we assess the impact of technology mediation on group conflict, we consider research on the use of communication technologies and, to the extent possible, information technologies that support collaborative work.

\section{Relational Outcomes}

For several decades, researchers have been concerned about the effect that interacting via technology has on group member relations, including cohesiveness, competition, group behaviors and attitudes, and group identity. One of the earliest theories explaining the relational effects of technology mediation is social presence theory, which argues that interpersonal and group processes are negatively affected when people interact over media that reduce their feeling of "being there" with their communication partners (Short et al. 1976). Social presence theory predicts that this reduced social presence will impair interpersonal relations.

Later work claims that mediation via technology reduces social cues and, because of their absence, alters the nature of group communication and group processes. With the potential for a reduction in critical contextual cues (e.g., status and gender), the social context may become less visible, causing people to display more disinhibited behaviors, to become less aware that they are engaged in social interaction, and to tend toward less consensus (Sproull and Kiesler 1991, Siegel et al. 1986). As a result of depersonalized interactions, groups communicating via technology are less likely to exchange relational information than face-to-face groups (Siegel et al. 1986). Other studies show that mediated groups are less cohesive than face-to-face groups (Straus and McGrath 1994), have lower group identity (Bouas and Arrow 1996), and exhibit more competitive behavior (Purdy et al. 2000). In short, mediated communication appears to negatively impact the ability of teams to build and maintain strong interpersonal relationships.

Differences among technologies in their ability to support communication have been explained by the media richness theory of Daft and Lengel (1984), which holds that media vary in the richness of the information transmitted and that richer media are more effective at reducing ambiguity and facilitating shared meaning (Daft et al. 1987). Media richness theory suggests that distributed team members might lessen the negative effects of mediation by choosing richer media.

The premises of social presence, reduced cues, and media richness theories are challenged by studies that suggest that time can remedy the relational problems that ensue from technology mediation. Many studies have found that mediated groups work more slowly than faceto-face groups (Walther and Burgoon 1992, Weisband 1992). In a meta-analysis of such studies, Walther et al. (1994) found that when task time limits were imposed on these more slowly paced groups, the groups exhibited lower socioemotional communication. When limits were expanded, relational outcomes often improved. There are caveats, however, to the benefits of time. Time does not universally improve socioemotional communication because not all groups are equally willing or able to develop relational closeness (Walther 1994). Also, we suspect that as team membership changes over time, a team's relational closeness must be reestablished as reconfigured teams learn anew how to communicate effectively via technology.

Social presence, reduced cues, and media richness theories also come under fire from scholars who decry the technological determinism these theories represent. Markus (1994a) noted two alternatives to technological determinism, both of which turn attention to technology users, their intentions, and the social context of technology use rather than the material characteristics of the 
technologies. The first alternative is the "rational-actor" perspective (Markus and Robey 1988, Kling 1980), which contends that individuals make choices about when and how to employ technologies. Research taking this perspective suggests that at times individuals desire and pursue the distance afforded by technology. They may choose, for example, to employ technology rather than talk face to face when interpersonal relationships are strained (Markus 1994a). A second alternative to technological determinism is the emergent-process view (Markus and Robey 1988, Pfeffer 1982), which holds that the effects of technology use are emergent and thus unpredictable. Given this, individuals may inadvertently worsen the impact of mediation by assuming they can prevent possible ill effects of technology use.

Recent extensions of Giddens' (1984) structuration theory also challenge the technological deterministic perspective by suggesting that people will engage in social practices that produce a particular structure of technology use, which may or may not be consistent with its intended use (Orlikowski 2000, DeSanctis and Poole 1994, Barley 1986). Structuration theory implies that distributed teams may alter their use of existing technologies in ways that will better serve the team. For example, there is evidence that teams find ways to share extensive and detailed information over e-mail systems (Hinds and Kiesler 1995, Lee 1994) and adapt technologies to improve their ability to coordinate better (Kraut et al. 1998).

No matter which theoretical perspective one takes, the anticipated effect of technology mediation on group conflict appears to be negative. The technological deterministic perspective suggests that distributed teams will experience greater opportunities for affective conflict as a result of technology mediation. Feelings of not "being there" with one's communication partners stand to prevent distributed team members from sharing relational information that help teams to develop trust. Fewer inhibited behaviors and a lower tendency for consensus may prompt affective conflict as team members neglect to censor their comments and fail to accommodate their team members' preferences. The rational-actor perspective highlights the potential for agency among distributed team members, but it fails to significantly lessen the negative implications of mediation because distributed team members, primarily lacking opportunities for face-to-face discourse, can choose only among technologies when wishing to communicate with one another. The rational-actor perspective provides hope, however, that for short periods and for specific purposes, choices that individuals make might deflect the onset of affective conflict. For example, Simons and
Peterson (2000) report that task conflict is more likely to lead to affective conflict when voices are raised, suggesting that distributed team members might opt for technologies like e-mail rather than the telephone for discussions in which task conflict is expected. This is consistent with empirical work suggesting that asynchronous communication mitigated negative interpretations of competitive behaviors, perhaps because these behaviors were obscured by the technology (MontoyaWeiss et al. 2001). The emergent-process perspective, which allows for unintended and unanticipated effects of technology use, provides little reason to suspect that technology mediation will not occasion conflict in distributed teams. Lee (1994), for example, illustrated that e-mail use is socially embedded and that e-mail users add meaning to the messages that they receive. However, the series of messages Lee examined also highlighted the potential for conflict, as Lee noted when describing the "politically sensitive and managerially troublesome meaning" (p. 153) occasioned by the initial e-mail. Finally, with regard to structuration theory, we concur with the conclusion of Kraut et al. (1998), that although users may modify technologies to suit their needs, technologies possess certain material limits that cannot be overcome. As evidence, Markus (1994b) showed that after the introduction of a new e-mail system, users felt that their interpersonal relationships were weakened even though they regularly used the telephone to "keep in touch." Although structuration theory implies that teams can limit the impact of technology on relational outcomes, the material properties of technologies, combined with the challenges of distance, render unlikely the possibility that distributed teams will be able to modify the technology adequately and consistently enough to match face-to-face communication.

In short, technology mediation engenders negative relational effects that we contend will precipitate affective conflict. These effects, including reduced cohesion and group identity, increased competitiveness, reduced consensus, and less sharing of relational information, contribute to lower trust, familiarity, and a sense of belonging on the team, and ultimately, we argue, induce affective conflict. Because the effects we have considered here concern group relations, we expect that their impact is primarily on affective conflict, although task and process conflict may be indirectly affected.

\section{Information Transfer}

Technology mediation also impacts information transfer among team members. Several problems related to information sharing and seeking emerge from the literature, including uneven distribution of information, unevenly 
weighted information, and information that resists transmission. Each problem portends negative effects with respect to conflict.

Uneven distribution can occur in at least two ways: Team members may be purposely or accidentally excluded from communications, or members may not reveal information that they uniquely hold. Work by Cramton (2001) highlights problems that can arise from exclusion. In her study of distributed student teams, not all members of the teams were copied on e-mails sent by one team member or another. As a result of this limited distribution, some team members worked with incomplete information while their colleagues assumed information had been universally shared. Conflict arose in the confusion that ensued. To complicate matters, team members attributed disagreements and miscommunications to individual-level factors rather than to the technology or the situation, a practice that further fueled interpersonal friction.

The technologies upon which distributed teams rely vary in the degree to which they promote inclusion and prevent exclusion. Audio- and videoconferencing, as well as Web-based meeting systems, support inclusion by allowing distant members to "attend" meetings, but they fail to prevent exclusion because they cannot guarantee that all members will be notified of the meeting. Additionally, Web-based systems like NetMeeting make it difficult for participants to keep track of who is remotely included in a meeting (Mark et al. 1999). Cramton's (2001) study reveals that technologies like e-mail, despite having features that support inclusion, may be more apt to facilitate exclusion of various team members, either through sender intent (acting as a rational actor) or mistake (in line with the emergent-process view of mediation). Her work points out the limitations of a purely technological determinist perspective by showing that even advanced features cannot ensure the uniform sharing of information.

Uneven distribution of information also results when team members fail to share uniquely held information. Information exchange is less complete and more biased in mediated groups as compared with face-to-face ones, and mediated groups are less likely to uncover information uniquely held by one member (Hollingshead 1996). Group members who rely on communication technologies find it more difficult to cue one another for the information they need and to interpret the cues being conveyed by other group members (Hollingshead 1996), which may be why unique information is not shared. The problem of incomplete information sharing, especially of uniquely held information, may be particularly detrimental to distributed teams because organizations often rely on such teams as a means of assembling expertise from a variety of distant locations.

Technology mediation also can impact the weight that various team members place on different pieces of information. Cramton (2001) noted that despite whatever importance a sender intended to attach to various topics within a single e-mail, team members often assigned different salience to the topics. As a result, some topics never received the attention the sender desired for them and at times were entirely overlooked, which led to frustration and misunderstanding.

Finally, some information is not readily transmitted via technology. Certainly some types of information, particularly those that can be digitized, can now be more easily transmitted via technologies such as file-transfer protocols and electronic workspaces, thus increasing the amount of this information that can be shared with distant teammates. However, many types of data continue to resist transmission via technology, with negative implications for conflict in distributed teams. This is particularly true in the case of contextual information that leads to shared awareness, such as who is in the office, what they are doing, what problems they are confronting, and the moment-to-moment social dynamics of the workplace. Weisband (2002) reported that although some mediated groups using a Web-conferencing system and e-mail were successful in conveying contextual information, many were not. Differences also exist in the degree to which technologies facilitate the exchange of contextual information (Olson and Olson 2000, Clark and Brennan 1991). As compared with the phone, which transmits little contextual information beyond background noises, newly developing awareness technologies that display information such as time zones, holidays, and current availability by type of medium for team members around the world are far better at aiding shared awareness (Atkins et al. 2002). Even with these technologies, considerable contextual information, such as unplanned actions that occur away from one's desk, remains untransmitted.

Research on technology mediation's information transfer effects suggests that distributed teams will be prone to conflict. Uneven distribution of information implies that team members will work and communicate on the basis of different information. As a result, they will be unlikely to recognize or resolve differences in perspectives, which have been shown to increase task conflict in groups with weak relationships (Brehmer 1976). Because technology impedes the ability of distributed teams to collect contextual information, these different perspectives may become entrenched, increasing the possibility of task conflict. Process conflict also 
is likely to arise when some members are excluded from messages. The progression of the team's work, seemingly purposeful and rational for members who receive all messages, may appear disjoint to those members who do not. Members who lack complete information may call into question the team's methods and trajectory. Finally, affective conflict, already engendered by mediation's relational effects, also may derive from information transfer effects. Uneven distribution of information, which can lead to frustration and misplaced blame, increases the potential for affective conflict.

\section{Coordination}

Coordination constitutes a third aspect of communication and collaborative work that is impaired by technology mediation. Purdy et al. (2000) reported that student groups working face to face collaborated more than distributed groups working over video, telephone, or chat. Moreover, collaborative efforts were less likely to be perceived in the mediated conditions. Field studies of distributed teams further suggest that technologies designed to increase shared awareness may exacerbate coordination problems. For example, an awareness technology that allowed team members to see what documents other team members had read resulted in team members opting not to read what others had in an effort to avoid duplication (Espinosa et al. 2000). Consequently, the team was limited in its ability to discuss materials.

Technology mediation also may induce time lags and sequencing problems that further hamper coordination. Cramton (2001) found that distributed teams using online chat to hold discussions with members around the globe experienced time lags that delayed some members' comments, rendering them "out of sync" with the larger conversation. With the sequence of responses disrupted, members may think their comments have been ignored, prompting frustration and irritation. Cramton's (2001) study also highlighted problems with asynchronous communication. Disparities in when messages were sent, received, and responded to resulted in individuals working with different information at different times.

The coordination problems imposed by technology mediation may precipitate conflict of all types. Process conflict is likely when the use of technologies renders some team members "out of sync" and makes it difficult to coordinate use of shared resources. Incompatibilities in work processes may give rise to disagreements about how work should get done. Coordination problems also may occasion task conflict, as can be expected when individuals have not examined the same materials as their colleagues or when they work on the basis of different information. Finally, the inability to coordinate work may lead to frustration and misconceptions, which in turn feed the potential for affective conflict.

\section{Overall Impact of Technology Mediation}

In short, notwithstanding the different affordances of technologies and the ability of users to make choices about how and when to use them, technology mediation has implications for conflict in distributed teams. Teams using the most advanced technologies experience difficulties, as do teams that have a host of technologies available to them. Just because one technology is found to be superior to another does not mean it is absent negative outcomes; research to date reveals that problems arise from nearly every available technology. The coordination problems we mention, for example, were uncovered with respect to computer conferencing and support systems, awareness technologies, e-mail, and shared workspaces. Moreover, advanced technologies are accompanied by their own litany of usability problems that compound the difficulties of mediation (see Fish et al. 1993, Gaver et al. 1992). Success of groupware tools, for instance, can be dependent on achieving a "critical mass" of users and on users' willingness to enter data into the system (Atkins et al. 2002). NetMeeting allows distant members to "attend" meetings, but Mark et al. (1999) found that many members could not participate because they were late in implementing the technology or had no one at their own site to consult with about the technology. Although technological advances may lessen a particular impact of mediation, it seems unlikely that they will ameliorate all such impacts and rather likely that they will occasion new ones. On these grounds, we propose that technology mediation engenders conflict of all types in distributed teams.

Proposition 5. As a result of the uneven information and difficulties in coordination that it occasions and its inability to transmit certain information, technology mediation engenders task conflict in teams.

Proposition 6. As a result of the negative relational effects, uneven information, unevenly weighted information, and difficulties in coordination that it occasions, technology mediation engenders affective conflict in teams.

PROPOSITION 7. As a result of the uneven information and difficulties in coordination that it occasions, technology mediation engenders process conflict in teams. 


\section{Summary of Conflict's Antecedents}

We consolidate our propositions on the antecedents and outcomes of group conflict in distributed teams into models for task, affective, and process conflict, respectively (see Figure 1). Perhaps the most striking result of our analysis is the number and variety of ways in which distance and technology mediation engender each type of conflict, as depicted by the multiple paths in each model.

\section{Conflict and Performance in Distributed Teams}

Having established that distributed teams are apt to experience a significant amount of conflict, we turn our attention to the consequences of conflict with respect to performance. Consistent with most of the existing literature on conflict and performance, we focus on team effectiveness and efficiency in performing tasks. We examine research on the impact of task, affective, and process conflict on performance in traditional teams and consider the implications for distributed teams.

\section{Task Conflict}

In general, conflict is detrimental to team performance. Task conflict, however, has been shown in many studies to have a positive relationship with performance (Eisenhardt et al. 1997, Jehn 1995), in part because groups consider more alternatives and think through options more thoroughly (Pelled et al. 1999). Considering diverse opinions and alternative strategies enables a group to avoid "groupthink" (Janis 1982) and arrive at better solutions (Pelled et al. 1999).

Although many studies have observed a positive effect, task conflict does not consistently lead to better performance. Recent studies of student project teams (Jehn et al. 1997), for example, have reported a negative relationship between task conflict and performance. Lovelace et al. (2001) reported that norms of openness and collaborative communication determined whether or not task conflict was beneficial. Recent research also has demonstrated that task conflict remains beneficial to teams only when it is not complicated by, and does not degenerate into, affective or process conflict (Jehn and Chatman 2000). Thus, although task conflict has the potential to be positive, research suggests it must be managed carefully through open, collaborative communication.

In contrast to what is found in traditional teams, we propose that the relationship between task conflict and performance will be consistently negative in distributed teams. We reason that task conflict is only beneficial to the extent that it is expressed and resolved through the process of building shared understanding. Sharing complex information and coming to consensus on even fairly mundane tasks is exceedingly difficult for teams separated by distance (Kraut et al. 2002). Communicating complex information via technology is more challenging because it takes longer (Straus and McGrath 1994), is subject to delay (Kraut et al. 1992), and can require more cognitive effort (Hinds 1999). Thus, distributed team members may have more difficulty engaging in collaborative interactions in which information must be shared and alternative perspectives understood. Overall, we expect that distributed teams will have difficulty resolving task conflict effectively and thereby will rarely gain its benefits. This prediction is supported by findings from Mortensen and Hinds (2001), who reported that task conflict was negatively related to performance in distributed product development teams.

Proposition 8. Task conflict detracts from performance in distributed teams.

\section{Affective Conflict}

Researchers have reported that affective conflict detracts from performance in student project teams (Jehn et al. 1997), R\&D teams (Evan 1965), work teams and management teams (Jehn 1997), and top-management teams (Eisenhardt et al. 1997, Amason 1996). Affective conflict often hampers performance as a result of the anxiety, hostility, and time and energy consumption associated with emotional disagreements (Pelled et al. 1999). Although a significant amount of research suggests a negative relationship between affective conflict and performance, there remains some ambiguity. Jehn (1995) observed that team members avoided other members with whom they did not get along. She posited that their evasion nullified the relationship between affective conflict and performance. Pelled et al. (1999) similarly surmised that subjects in the project teams they studied found ways to cope with individuals with whom they had affective conflicts, thereby preventing a negative impact on performance. These studies suggest that it is not affective conflict itself, but the open expression of affective conflict, that contributes to a negative conflict-performance relationship.

We propose that affective conflict will continue to detract from performance in distributed teams for the same reasons that it often does in traditional teams. We further note that in distributed teams, the opportunity to avoid affective conflict may be higher because team members do not encounter each other regularly throughout the day-They can often go days, if not weeks, 
Figure 1 Antecedents of Conflict on Distributed Teams

(a) Antecedents of task conflict

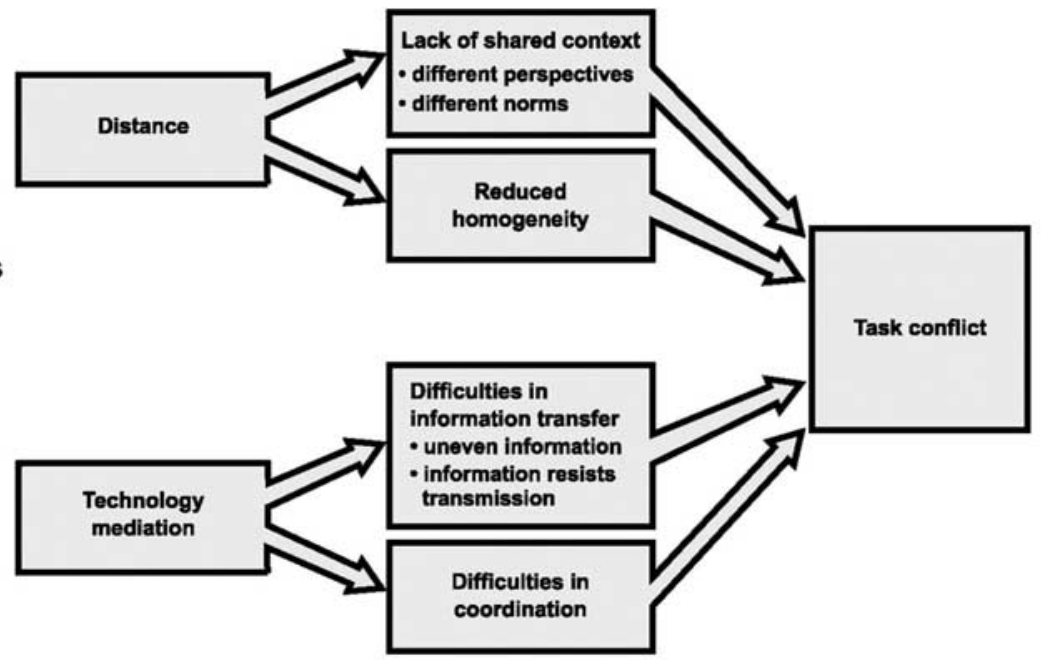

(b) Antecedents of affective conflict

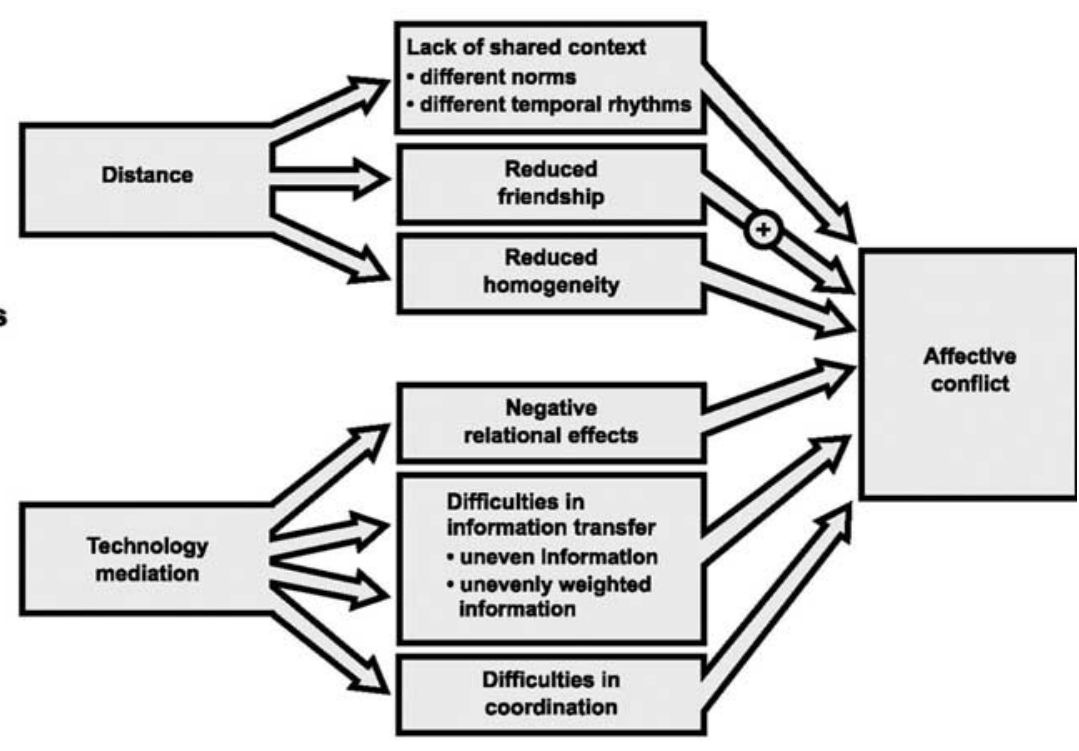

(c) Antecedents

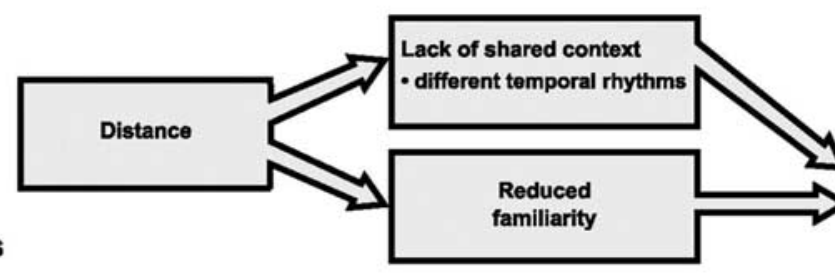
of process conflict

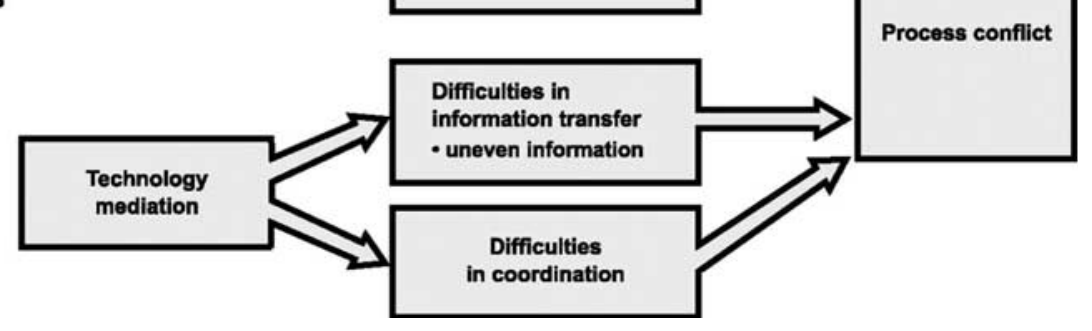


without "speaking" to a distant team member. Similarly, opportunities for open expression of affective conflict may be lower because mediating technologies provide less-satisfying ways of discussing emotional topics. These factors point toward less destructive affective conflict in distributed teams. We argue, however, that members of distributed teams will be prone to more faulty and harsh attributions (Cramton 2002) and that such attributions will counterbalance the benefits of avoidance. We therefore posit that affective conflict will have an overall detrimental effect on performance in distributed teams.

Proposition 9. Affective conflict detracts from performance on distributed teams.

\section{Process Conflict}

The few studies that have examined the relationship between process conflict and performance on traditional teams suggest that process conflict, like affective conflict, generally has negative effects (Jehn and Mannix 2001, Jehn 1997). Process conflict appears to detract from performance because effort is absorbed by disagreements, and inefficiencies result from confusion about resources and responsibilities (Jehn 1997). As with affective conflict, we argue that process conflict will detract from performance in distributed teams for the same reasons that it does on traditional teams. In fact, in distributed teams, confusion about resources and responsibilities may be even more detrimental and take more time to resolve because of divergent perspectives and communication challenges. This conclusion is consistent with the observations made by Grinter et al. (1999), who noted severe inefficiencies and errors in distributed teams attempting to coordinate their work in the face of incompatible work processes.

Proposition 10. Process conflict detracts from performance in distributed teams.

\section{Conflict as a Dynamic Process}

With time, team members may get to know one another better, learn how to work together more effectively, and develop ways to best employ technology. Models of group conflict that fail to take into account how teams evolve over time run the risk of being incomplete, and possibly misleading. Thus, we complete our model of conflict and performance on distributed teams by adding dynamic components. In the interest of providing insights into how teams might mitigate the problems of distribution, we discuss the proposed moderators in terms of their potential for positive effects. A reduction or absence of these moderators, however, will be equally likely to exacerbate conflict and its negative effects.

To build our model, we examine three aspects of dynamic behavior with respect to conflict in distributed teams: how teams may mitigate the negative effects of distance and technology mediation, factors that might moderate the effects of distance and technology mediation on conflict, and the role of conflict handling over time. Because the literature related to the dynamics of group conflict is not as robust as that concerning antecedents to conflict, our arguments here are more speculative. Our dynamic model is represented in Figure 2.

\section{Preventative Measures}

Teams may be able to mitigate the negative effects of distance and use of mediating technologies in at least five ways. Perhaps the most immediate way to diminish the negative effects of distance is to dislodge, if only temporarily, distance itself; for example, by increasing the frequency and length of face-to-face meetings (see Kraut et al. 1992). Because face-to-face interaction facilitates interpersonal relationships, more face-to-face meetings should promote more familiarity and friendship. At the extreme, organizations can collocate team members for a period of time, which should enhance shared context.

Purposely conveying contextual information when working remotely is another way that teams may mitigate the ill effects of distance. Team members might make it a point to share information about vacation schedules, office politics, and so forth. They also might employ new awareness technologies to automate the process of sharing some contextual information (see Atkins et al. 2002). By making a special effort to convey contextual information, team members improve the extent to which their teammates understand their context and increase opportunities to build familiarity and friendship.

A third way that teams and organizations can mitigate the impact of distance, particularly on shared contexts, is by creating similar contexts at different sites. Although there are many subtle yet important contextual elements that cannot be standardized and others that, once made similar, diverge due to local events and pressures, some standardization of work processes, tools, and systems can help mitigate conflict in distributed teams. Dissimilar work processes create an occasion for conflict as team members struggle to integrate across different technologies, understand nonstandard formats, and negotiate differences. For example, if a software development team is using different compilers across two distant sites, conflict will likely emerge because shared code cannot 
Figure 2 Dynamic Model of Conflict and Performance on Distributed Teams

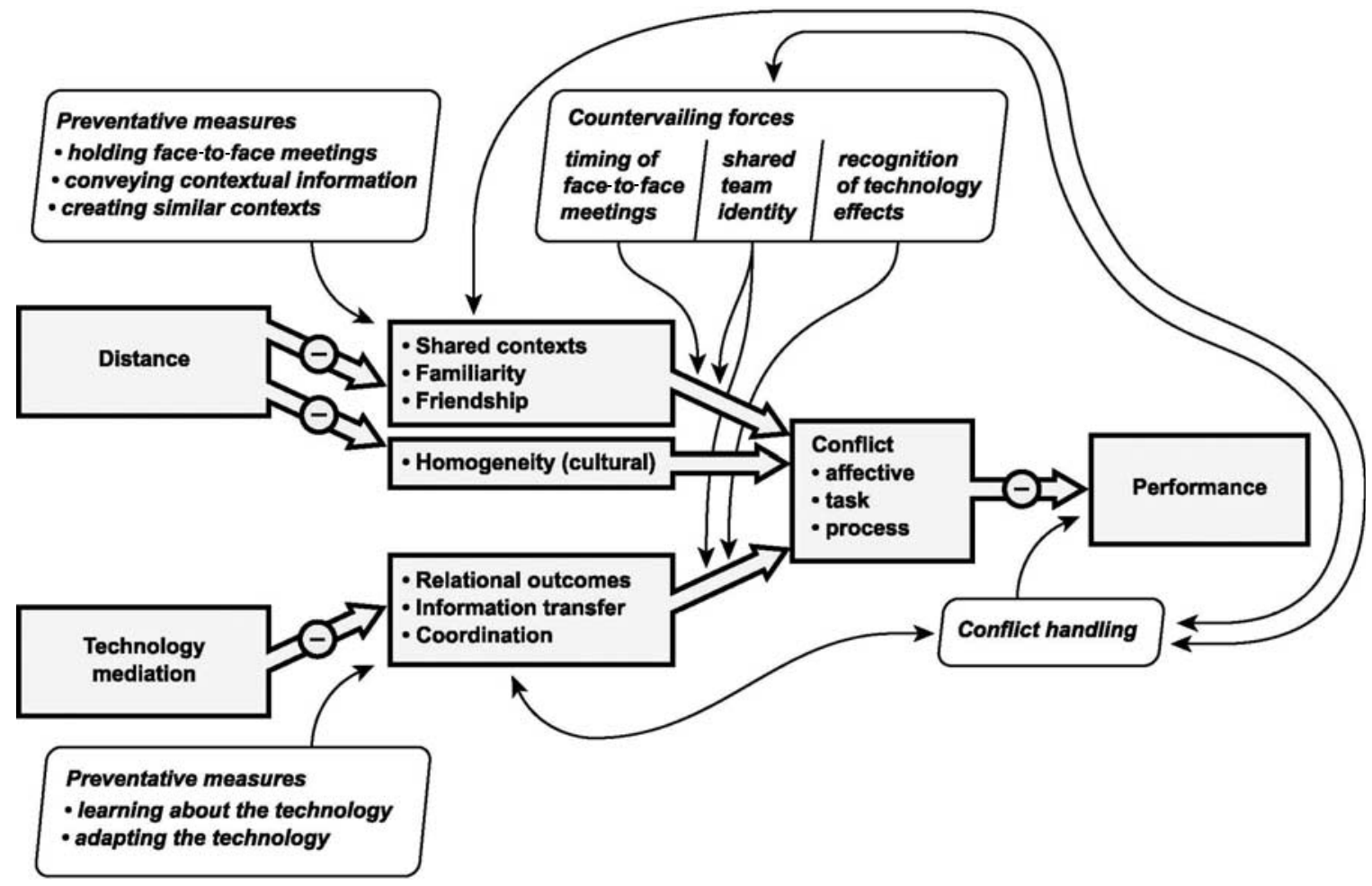

be tested easily or adequately. In this case, using a single compiler could ease coordination problems faced by the team.

Distributed teams also may dampen some of the negative effects of technology mediation. Over time, team members may adapt to the communication and information technologies that they employ through training and use or by altering the technology to meet their needs. As discussed earlier, structuration theory suggests that people will engage in social practices that produce a particular structure of technology use (Orlikowski 2000, DeSanctis and Poole 1994, Barley 1986). These patterns of use may change over time, sometimes as a result of user training. As evidence, Orlikowski (2000) observed that teams with higher levels of interest in Lotus Notes and higher levels of knowledge regarding its properties and functionalities were more effective in using it. Without training and confidence in the technology, people may avoid tools, thus severely limiting the flow of information among distant sites (Olson and Teasley 1996). In short, as teams learn more about the technologies they use, they will be better able to communicate, share information, and coordinate.

We also have reason to suspect that, over time, distributed teams may change the communication technologies they employ in ways that will better serve the team.
Longitudinal studies report that groups adapt communication technologies to good effect (Walther and Burgoon 1992, Chidambaram 1989), for example, altering the technology to improve coordination (Kraut et al. 1998). Team members also choose technologies that they feel are more appropriate (Hinds and Kiesler 1995, Markus 1994b) and use technology to enact social processes that better fit their needs (DeSanctis and Poole 1994, Orlikowski 2000). When team members elect to use technologies that more effectively convey the affect and information demanded at the time, they may mitigate the effect of mediating technologies. For example, by choosing to make periodic telephone calls to keep in touch rather than relying exclusively on e-mail, team members may facilitate better relational outcomes (see Markus 1994a). As this happens, distributed teams should exhibit fewer immediate outcomes of distribution and, therefore, less conflict of all types.

We have argued that teams can use preventative measures to mitigate many of the negative effects of distance and technology mediation as they meet face to face, learn more about one another's work environments, create similar contexts, and learn about and adapt the technologies on which they rely. These negative effects, however, will never entirely disappear. Although distance can be suspended during face-to-face meetings, 
when team members return to their respective sites distance will again exist among them. As a result, the negative effects of distance will again accrue. Similarly, although some context can be shared by visiting one another's sites, sharing contextual information, and adopting similar processes, contexts change over time and gaps will inevitably remain. Limits also exist with respect to learning to use mediating technologies, adapting technologies to better serve the team, and choosing more appropriate technologies for a given task. Technologies change and have material limits that are difficult, if not impossible, to overcome. We therefore argue that the preventative measures taken by teams may moderate, but cannot eliminate, the negative effects of distance and mediating technologies. We also suggest that teams that do not learn, do not adapt technologies to better serve their purposes, and are not thoughtful with regard to choosing technologies are likely to experience more severe negative outcomes associated with distance and technology mediation.

\section{Countervailing Forces}

To the extent that the immediate outcomes of distribution cannot be prevented, they will have an impact on group conflict. However, several countervailing forcesincluding shared team identity, the timing of face-to-face meetings, and recognition of technology effects-will moderate this impact.

Over time, effective teams generate a shared team identity. Teams with a strong team identity see themselves as a unit working toward a common goal and may have more integrative communications (see Maznevski 1994). We, along with others (Mannix et al. 2002, Mortensen and Hinds 2001), argue that a shared team identity helps distributed teams bridge distance by creating a psychological tie among team members. In the absence of a team identity, team members may not see themselves as a cohesive unit, may have less faith in the behaviors and intentions of other members, and may be less likely to talk through issues that arise. At the extreme, team members may see themselves as occupying different social categories or groups, resulting in distorted judgments about other group members (Pelled and Adler 1994). These perceptions of "otherness" within a group can make conflict more difficult to resolve (Williams and O'Reilly 1998) because team members do not give others the "benefit of the doubt."

The timing of face-to-face interaction also may be an important countervailing force in limiting the effects of distance and mediating technologies. Distributed teams appear to gain more if they meet early in the development of the group (Kraut et al. 1992), enabling members to form relationships that can be supported over technologies (Armstrong and Cole 2002). For distributed teams with clear deadlines or a disbanding point, other times also appear deserving of face-to-face meetings. Critical hand-off points, for example, are likely candidates for face-to-face meetings of team members, including the final integration and conclusion of the work because of the potential for misunderstandings and the need for collaborative problem solving (see Grinter et al. 1999). If distributed teams are able to meet face to face at the points with the most potential for conflict, then conflict may be reduced or diminished.

The final countervailing force is the team's experience with technology, particularly the extent to which the team is able to recognize the effect of their technology use on relational outcomes, information transfer, and coordination within the team. When team members recognize the limitations of the technologies-for instance, that e-mail messages can seem unintentionally harsh and that addressing features may cause team members to be inadvertently left out of important communicationsthey are more likely to give team members the benefit of the doubt and investigate issues before conflict ensues. In these cases, communication can halt misattributions and escalating conflict (Jarvenpaa and Leidner 1999).

\section{Conflict-Handling Strategies}

In addition to preventative measures and countervailing forces, conflict-handling strategies play an important role in the dynamics of conflict. The severity and recurrence of conflict is determined, in part, by the extent to which each event is resolved to the satisfaction of all parties. Even with severe conflicts, healthy and open resolution of the conflict will breed trust and ease contentiousness as new sources of conflict arise (see Lovelace et al. 2001). A number of researchers have used Thomas' (1992) two-dimensional taxonomy of conflict-handling intentions to understand how conflict is resolved in teams. Thomas argued that when both parties are attempting to maximize their own interests (assertiveness) as well as the other party's interests (cooperativeness), they have a "collaborative" intent. In the absence of a collaborative intent, one of four alternative approaches are used: avoidance, competition, compromise, or accommodation. Each of these approaches, Thomas argued, leads to one or both parties ultimately frustrated with the outcome. We posit that distant team members will have no more or less collaborative intent 
than collocated team members. Establishing collaborative norms, however, may be significantly more difficult in distributed teams. Collaborative norms of conflict handling improve performance, whereas avoidance and compromise detract from it (Montoya-Weiss et al. 2001, Xie et al. 1998). To be effective, parties must work to resolve the underlying interests of each party. Doing so requires sharing information about each party's interests and engaging in discussion and brainstorming to discover innovative ways to create a win-win solution (Lovelace et al. 2001, Tinsley 1998).

In distributed teams, collaborative conflict handling may be fraught with difficulty. Thomas (1992) held that trust, positive mutual regard, mutual attraction, cohesiveness, and adequate opportunities to interact (among others) are crucial for fostering collaboration. Some of these factors, however, are directly affected by distance and technology mediation. Distance diminishes the development of friendships among team members (which is akin to attraction and cohesiveness), often undermines trust, and reduces opportunities to interact, especially on an informal basis. Technology mediation also threatens a team's ability to share information, which is a necessary condition for collaborative work (see Kraut et al. 2002) and is crucial for integrative conflict resolution (Tinsley 1998). Consistent with this, Purdy et al. (2000) reported that participants in a negotiation over mediated technologies as compared with face to face engaged in less collaborative conflict resolution. Thus, distributed teams may be unable to manifest their collaborative intent and be reduced to relying on other, less effective conflicthandling strategies.

With effort, some teams can overcome this challenge and handle conflict collaboratively. In these cases, we predict that they will not only be more effective at resolving issues, but also will accrue interpersonal and coordination benefits from collaborative conflict handling (see Lovelace et al. 2001). They will increase their familiarity, friendship, relational outcomes, ability to share information, and ability to collaborate and coordinate. Future conflicts may be reduced (see Thomas 1992). Conflict-handling norms also may contribute to shared team identity. When teams deal with conflict collaboratively, they are likely to establish stronger bonds and have more integrated goals. In sum, healthy conflicthandling norms will lessen the impact that conflict has on performance and reduce the potential for future conflict.

\section{Overall Dynamics of Conflict}

Along with others (e.g., Jehn and Mannix 2001), we argue that conflict in teams is a complex, dynamic pro- cess that changes over time and is impacted by a variety of factors. In the course of this process, teams may be able to mitigate many of the negative effects of distance and reliance on mediating technologies. As a result, conflict in teams may diminish over time, but only to the extent that teams are aware of and mindful of the changes they are making. Untended, shared identity will likely diminish rather than increase in distributed teams (Mannix et al. 2002). Face-to-face meetings also are likely to fall by the wayside as schedules begin to slip and budgets become tight. Further, conflict handling is more likely to evolve into avoidance if healthy norms are not established and maintained. We argue that teams must be cognizant of and attentive to these preventative measures and countervailing forces to sustain a healthy amount of conflict and to improve over time.

Proposition 11. Distributed teams moderate the negative effects of distance by meeting face to face, purposely conveying contextual information, and creating similar contexts at distant sites.

Proposition 12. Distributed teams moderate the negative effects of mediating technology by learning about and adapting the technologies they employ.

Proposition 13. In distributed teams, shared identity and well-timed face-to-face meetings moderate all of the immediate impacts of distance on conflict.

Proposition 14. In distributed teams, shared identity and recognition of the effects of technology moderate all of the immediate impacts of technology mediation on conflict.

Proposition 15. Distributed teams are less likely than traditional teams to engage in collaborative conflict handling.

Proposition 16. In distributed teams, the approach used for handling conflict will impact and be impacted by shared team identity and all of the immediate outcomes of distribution.

Proposition 17. Conflict handling in distributed teams moderates the conflict-performance relationship such that more collaborative conflict handling reduces the negative effects of conflict on performance.

\section{Discussion}

Our analysis provides the beginnings of a comprehensive explanation for the high levels of conflict that have been observed in empirical studies of distributed teams. A key aspect of our analysis is the teasing out of antecedents that derive from distance separately from 
those that derive from technology mediation. The latter may be experienced by traditional teams to the extent that their members rely on technology to communicate and work with one another even when collocated; the former, however, are likely to be unique to distributed teams. Because only one of the many antecedents we identify is expected to reduce conflict, whereas all of the others are expected to increase it, our analysis helps to explain why distributed teams are experiencing high levels of conflict.

The antecedents we have identified exist in addition to the established causes of conflict that affect teams of all types. We suspect that other established antecedents (e.g., cross-functional composition and tenure diversity; see Pelled et al. 1999) will have similar effects on distributed teams as they have on traditional teams. We also suspect that distributed as compared with traditional teams may experience different levels of these antecedents. For example, distributed teams may have higher functional diversity as organizations strive to assemble diverse expertise from multiple sites, and less continuity of membership as organizations dynamically allocate team members across projects based on changing demands (DeSanctis and Monge 1999). To the extent that our suspicion bears out, distributed teams as compared with traditional teams may face even higher levels of conflict.

Technology mediation, along with its limitations, enables forms of communication that are not otherwise feasible or possible. Team members can send messages one day and wait for a response the next, messages can follow a person around the globe, and communication can be easily archived for later reference. From our analysis, however, we have no reason to believe that the benefits that can result from technology use will enable distributed teams to prevent the conflict that we anticipate. Still, these benefits make distributed work possible and may alter it in ways quite different from collocated work, therefore providing an important opportunity for continued research.

New technologies bring with them advanced features and new affordances. Given that distributed teams must rely heavily upon communication and information technologies, it is worth considering the extent to which our conclusions are contingent upon the current state of technology. Certainly, shared awareness technologies (and more recently, immersive environments; see Blascovich et al. 2002) are making considerable strides in conveying contextual information across distances. However, the unanticipated consequences and usability problems that we have noted suggest that the problems of mediation will be with us for some time. Additionally, some aspects of face-to-face communication that serve to prevent conflict may never be achieved by technologies, which have limited capacity for capturing and transmitting the myriad actions that occur simultaneously in any conversation or meeting.

Our analysis, however, does provide implicit recommendations for the kinds of features that new technologies might incorporate (as some already do) to mitigate the kinds of misunderstandings that lead to conflict. Such features include automatic inclusion of all team members on messages, mechanisms for indicating the importance of items within messages, and passive provision of contextual information. Our conclusions also suggest protocols with regard to current technologiesincluding practices for shared document retrieval and storage and acknowledgment of local conditions within messages - that might cue distributed team members for the kinds of effects to anticipate when using new technologies.

Our models are incomplete in a number of respects, which we note here to acknowledge limitations and to suggest future theoretical work. One major absence is an exploration of the role that established moderators of conflict's impact on performance might play in the case of distributed teams. Task interdependence is a prime example: It consistently moderates the relationship between all types of conflict and performance on traditional teams. We also did not consider a measure of geographic distribution or team size, even though it seems reasonable to expect that a team spanning 20 sites might experience more negative impacts than a team spanning only 2 sites. Similarly, our conclusions are likely to differ for teams with only a single member at each site versus those with multiple members at each site. We restricted ourselves to geographically distributed teams, ignoring other types of distributed teams (such as those with members from various organizations) whose experiences with conflict may differ significantly. Finally, we did not consider different types of teams (e.g., project teams versus management teams) or factors associated with task (e.g., type, complexity, and routineness). We suspect that future investigations of the factors we list here will make the model more comprehensive and allow more precise predictions.

More research also is needed on information technologies and information transfer. Studies have shown that uneven information can result from exclusion of team members on messages or from failure of team members to share uniquely held information; studies further show that uneven information prompts task conflict. Task conflict may emerge because some team members are operating with incomplete information, suggesting 
that once the information is provided, agreement will quickly result and resolution of the conflict will be swift. Alternatively, task conflict may arise because the team as a whole is unable to resolve differing perspectives when some members are operating without certain information. In this case, team members must comprehend, translate, and incorporate the missing information, once provided, in a much more substantive, time consuming, and perhaps collaborative manner. Should the former situation hold sway, technology features that promote inclusion may remedy much of the problem. In the latter situation, mere provision is insufficient. Research that investigates the nature of information that tends to be unevenly distributed across a team and how members use the information that they possess would provide a more complete picture of the impact of information transfer difficulties on conflict.

As we have noted, time plays an important role for the relational outcomes of technology mediation. In addition, it lies at the heart of the emergent-process perspective of technology's impact and is implicit in many technology structuration arguments. Existing longitudinal studies demonstrate that conflict occurs in teams and that how it is handled over time matters (Jehn and Mannix 2001). Thus, it is important that future empirical studies examine the role of time and the dynamics that it fosters. Interviews and surveys will aid investigations of conflict in distributed teams, but observations may be necessary to capture the details of how team members provoke, engage in, resolve, and strive to prevent conflict. Because distributed teams by definition span sites, observational studies will require teams of likewise-distributed researchers. A complementary strategy could include the collection of electronic communication and information exchanged via technology. Such data, particularly if collected over time, could provide a critical window into the ongoing dynamics of distributed teams. The study of conflict in distributed teams thus appears to necessitate multiple-method large-scale longterm studies, studies that will be ambitious in their goals but that stand to yield important findings that will inform theory, managerial practice, and technology design.

\section{Acknowledgments}

This research was partially supported by National Science Foundation Grants IIS-9872996 and DMI-9996081 to the first and second authors, respectively.

\section{References}

Amason, A. C. 1996. Distinguishing the effects of functional and dysfunctional conflict on strategic decision making: Resolving a paradox for top management teams. Acad. Management J. 39 123-148.
Ancona, D. G., C.-L. Chong. 1996. Entrainment: Pace, cycle, and rhythm in organizational behavior. Res. Organ. Behavior 18 251-284.

Armstrong, D. J., P. Cole. 2002. Managing distances and differences in geographically distributed work groups. P. J. Hinds, S. Kiesler, eds. Distributed Work. MIT Press, Cambridge, MA, 167-186.

Atkins, D., D. Boyer, M. Handel, J. Herbsleb, A. Mockus, G. Wills. 2002. Achieving speed in globally distributed project work. Paper presented at the Human Comput. Interaction Consortium, Winterpark, CO.

Barley, S. R. 1986. Technology as an occasion for structuring: Evidence from observation of CT scanners and the social order of radiology departments. Admin. Sci. Quart. 31 78-108.

Blascovich, J., J. Loomis, A. C. Beall, K. R. Swinth. 2002. Immersive virtual environment technology as a research tool for social psychology. Psych. Inquiry 13 103-125.

Bouas, K., H. Arrow. 1996. The development of group identity in computer and face-to-face groups with membership change. Comput.-Supported Cooperative Work 4 153-178.

Brehmer, B. 1976. Social judgment theory and the analysis of interpersonal conflict. Psych. Bull. 83 985-1003.

Chidambaram, L. 1989. Relational development in computersupported groups. MIS Quart. 20 143-163.

Clark, H. H., S. E. Brennan. 1991. Grounding in communication. L. Resnick, J. Levine, S. Teasley, eds. Perspectives on Socially Shared Cognition, American Psychological Association, Washington, D.C., 127-149.

Cohen, S. G., D. E. Bailey. 1997. What makes teams work: Group effectiveness research from the shop floor to the executive suite. J. Management 23(3) 239-290.

Cramton, C. D. 2001. The mutual knowledge problem and its consequences for dispersed collaboration. Organ. Sci. 12(3) 346-371. 2002. Attribution in distributed work groups. P. J. Hinds, S. Kiesler, eds. Distributed Work. MIT Press, Cambridge, MA, 191-212.

Daft, R. L., R. H. Lengel. 1984. Information richness: A new approach to managerial behavior and organization design. Res. Organ. Behavior 6 191-233.

$\ldots$, _, L. K. Trevino. 1987. Message equivocality, media selection, and manager performance: Implications for information systems. MIS Quart. 11 355-366.

DeSanctis, G., P. Monge. 1999. Introduction to the special issue: Communication processes for virtual organizations. Organ. Sci. 10 693-703.

_ M. S. Poole. 1994. Capturing the complexity in advanced technology use: Adaptive structuration theory. Organ. Sci. 5 121-147.

Deutsch, M. 1969. Conflicts: Productive and distructive. J. Soc. Issues 25 7-41.

Eisenhardt, K. M., J. L. Kahwajy, L. J. Bourgeois. 1997. Conflict and strategic choice: How top management teams disagree. California Management Rev. 39(2) 42-62.

Espinosa, A., J. Cadiz, L. Rico-Gutierrez, G. Lautenbacjer, R. Kraut, L. Scherlis. 2000. Coming to the wrong decision quickly: Why awareness tools must be matches with appropriate tasks. Proc. Human Factors Comput. Systems, CHI'2000, ACM Press, New York, 392-399. 
Evan, W. 1965. Conflict and performance in R\&D organizations. Indust. Management Rev. 7 37-46.

Festinger, L., S. Schacter, K. Bach. 1950. Social Pressures in Informal Groups. Stanford University Press, Palo Alto, CA.

Fish, R. S., R. E. Kraut, R. Root, R. E. Rice. 1993. Video as a technology for informal communication. Comm. ACM 36 8-61.

Fussell, S. R., R. M. Krauss. 1992. Coordination of knowledge in communication: Effects of speakers' assumptions about what others know. J. Personality Soc. Psych. 62(3) 378-391.

Gaver, W. W., T. Moran, A. MacLean, L. Lovstrand, P. Dourish, K. A. Carter, W. Buxon. 1992. Realizing a video environment: EuroPARC's RAVE system. Proc. ACM Conf. Human Factors Comput. Systems (CHI'92).

Giddens, A. 1984. The Constitution of Society: Outline of the Theory of Structure. University of California Press, Berkeley, CA.

Goodman, P. S., D. P. Leyden. 1991. Familiarity and group productivity. J. Appl. Psych. 76 578-586.

Grinter, R. E., J. D. Herbsleb, D. E. Perry. 1999. The geography of coordination: Dealing with distance in R\&D work. Paper presented at the 1999 SIGGROUP Conference, Phoenix, AZ.

Hackman, J. R. 1987. The design of work teams. J. W. Lorsch, ed. Handbook of Organizational Behavior. Prentice-Hall, Englewood Cliffs, NJ, 315-342.

Hinds, P. J. 1999. The cognitive and interpersonal costs of video. Media Psych. 1 283-311.

_ S. Kiesler. 1995. Communication across boundaries: Work, structure, and the use of communication technologies in a large organization. Organ. Sci. 6 373-393.

Hollingshead, A. B. 1996. Information suppression and status persistence in group decision making: The effects of communication media. Human Comm. Res. 23(2) 193-219.

Janis, I. L. 1982. Victims of Groupthink. Houghton Mifflin, Boston, MA.

Jarvenpaa, S., D. Leidner. 1999. Communication and trust in global virtual teams. Organ. Sci. 10 791-815.

Jehn, K. A. 1995. A multimethod examination of the benefits and detriments of intragroup conflict. Admin. Sci. Quart. 40 256-282.

1997. A quantitative analysis of conflict types and dimensions in organizational groups. Admin. Sci. Quart. 42(3) 530-558.

_ J. A. Chatman. 2000. The influence of proportional and perceptual conflict composition on team performance. Internat. J. Conflict Management 11 56-73.

_ E. A. Mannix. 2001. The dynamic nature of conflict: A longitudinal study of intragroup conflict and group performance. Acad. Management J. 44(2) 236-251.

— C. Chadwick, S. M. B. Thatcher. 1997. To agree or not to agree: The effects of value congruence, individual demographic dissimilarity, and conflict on workgroup outcomes. Internat. J. Conflict Management 8 287-305.

Karnoe, P. 1995. Competence as process and the social embeddedness of competence building. Acad. Management Best Paper Proc., Vancouver, Academy of Management, Briarcliff Manor, NY, 427-431.
Kiesler, S., J. N. Cummings. 2002. What do we know about proximity and distance in work groups? A legacy of research. P. J. Hinds, S. Kiesler, eds. Distributed Work. MIT Press, Cambridge, MA, $57-80$.

Kling, R. 1980. Social analyses of computing: Theoretical perspectives in recent empirical research. ACM Comput. Surveys 12 61-110.

_ R. E. Rice, C. Cool, R. S. Fish. 1998. Varieties of social influence: The role of utility and norms in the success of a new communication medium. Organ. Sci. 9(4) 437-453.

Kraut, R. E., R. S. Fish, B. Chalfonte. 1992. Requirements and media choice in collaborative writing. Human Comput. Interaction 7 375-407.

_ , S. R. Fussel, S. E. Brennan, J. Seigel. 2002. Understanding effects of proximity on collaboration: Implications for technologies to support remote collaborative work. P. J. Hinds, S. Kiesler, eds. Distributed Work. MIT Press, Cambridge, MA, 137-162.

Lee, A. S. 1994. Electronic mail as a medium for rich communication: An empirical investigation using hermeneutic interpretation. MIS Quart. 18(June) 143-157.

Lovelace, K., D. L. Shapiro, L. R. Weingart. 2001. Maximizing cross-functional new product teams' innovativeness and constraint adherence: A conflict communications perspective. Acad. Management J. 44 779-783.

Mannix, E. A., T. Griffith, M. A. Neale. 2002. The phenomenology of conflict in distributed work teams. P. J. Hinds, S. Kiesler, eds. Distributed Work. MIT Press, Cambridge, MA, 213-233.

Mark, G., J. Grudin, S. E. Poltrock. 1999. Meeting at the desktop: An empirical study of virtually collocated teams. Proc. ECSCW'99, The $6^{\text {th }}$ Eur. Conf. Comput. Supported Cooperative Work, Copenhagen, Denmark (September).

Markus, M. L. 1994a. Finding a happy medium: Explaining the negative effects of electronic communication on social life and work. ACM Trans. Inform. Systems 12 119-149.

1994b. Electronic mail as the medium of managerial choice. Organ. Sci. 5 502-527.

— D. Robey. 1988. Information technology and organizational change: Causal structure in theory and research. Management Sci. 34(5) 583-598.

Maznevski, M. L. 1994. Understanding our differences: Performance in decision-making groups with diverse members. Human Relations 47(5) 531-552.

, K. M. Chudoba. 2000. Bridging space over time: Global virtual team dynamics and effectiveness. Organ. Sci. 11 437-492.

Montoya-Weiss, M. M., A. P. Massey, M. Song. 2001. Getting it together: Temporal coordination and conflict management in global virtual teams. Acad. Management J. 44 1251-1262.

Mortensen, M., P. J. Hinds. 2001. Conflict and shared identity in geographically distributed teams. Internat. J. Conflict Management 12(3) 212-238.

Murnighan, J. K., D. J. Conlon. 1991. The dynamics of intense work groups: A study of British string quartets. Admin. Sci. Quart. 36 165-186.

Olson, G. M., J. S. Olson. 2000. Distance matters. Human Comput. Interaction 15 139-179. 
Olson, J. S., S. Teasley. 1996. Groupware in the wild: Lessons learned from a year of virtual colocation. Proc. Conf. Comput.-Supported Cooperative Work, The Association for Computing Machinery, New York, 419-427.

O’Reilly, C., K. Williams, S. Barsade. 1997. Group demography and innovation. Does diversity help? E. Mannis, M. Neale, eds. Research in the Management of Groups and Teams, Vol. 1. JAI Press, Greenwich, CT.

Orlikowski, W. 1992. The duality of technology: Rethinking the concept of technology in organizations. Organ. Sci. 3(3) 398-427.

_ 2000. Using technology and constituting structures: A practice lens for study technology in organizations. Organ. Sci. 11 404-428.

Pelled, L. H. 1996. Demographic diversity, conflict, and work group outcomes: An intervening process theory. Organ. Sci. 7 615-631.

— , P. S. Adler. 1994. Antecedents of intergroup conflict in multifunctional product development teams: A conceptual model. IEEE Trans. Eng. Management 41(1) 21-28.

_ K. K. M. Eisenhardt, K. R. Xin. 1999. Exploring the black box: An analysis of work group diversity conflict and performance. Admin. Sci. Quart. 44 1-28.

Pfeffer, J. 1982. Organizations and Organization Theory. Pitman, Marshfield, MA.

Purdy, J. M., P. Nye, P. V. Balakrishnan. 2000. The impact of communication media on negotiation outcomes. Internat. J. Conflict Management 11 162-187.

Schober, M. F. 1998. Different kinds of perspective-taking. S. Fussell, R. Krauss, eds. Social and Cognitive Approaches to Interpersonal Communication. Lawrence Erlbaum, Mahwah, NJ.

Shah, P. P., K. A. Jehn. 1993. Do friends perform better than acquaintances? The interaction of friendship, conflict, and task. Group Decision Negotiation 2 149-165.

Short, J., E. Williams, B. Christie. 1976. The Social Psychology of Telecommunications. John Wiley \& Sons, London, U.K.

Siegel, J., V. Dubrovsky, S. Kiesler, T. W. McGuire. 1986. Group processes in computer-mediated communication. Organ. Behavior Human Decision Processes 37 157-187.

Simons, T. L., R. S. Peterson. 2000. Task conflict and relationship conflict in top management teams: The pivotal role of intragroup trust. J. Appl. Psych. 85 102-111.

Sproull, L., S. Kiesler. 1991. Connections: New Ways of Working in the Networked Organization. MIT Press, Cambridge, MA.

Straus, S., J. E. McGrath. 1994. Does the medium matter? The interaction of task type and technology on group performance and member reactions. J. Appl. Psych. 79 87-97.

Thomas, K. W. 1992. Conflict and negotiation processes in organizations. M. D. Dunnette, L. M. Hough, eds. Handbook of Industrial and Organizational Psychology, Vol. 3. Consulting Psychologists Press, Palo Alto, CA, 651-717.

Tinsley, C. 1998. Models of conflict resolution in Japanese, German and American cultures. J. Appl. Sci. 83 316-323.

Tyre, M., E. von Hippel. 1997. The situated nature of learning in organizations. Organ. Sci. 8 71-83.

Walther, J. B. 1994. Anticipated ongoing interaction versus channel effects on relational communication in computer-mediated interaction. Human Comm. Res. 20 473-501.

- J. Burgoon. 1992. Relational communication in computermediated interaction. Human Comm. Res. 19 50-88.

- J. F. Anderson, D. Park. 1994. Interpersonal effects in computer-mediated interaction: A meta-analysis of social and anti-social communication. Comm. Res. 21 460-487.

Weisband, S. 1992. Group discussion and first advocacy effects in computer-mediated and face-to-face decision making groups. Organ. Behavior Human Decision Processes 53 352-380.

—. 2002. Maintaining awareness in distributed team collaboration: Implications for leadership and performance. P. J. Hinds, S. Kiesler, eds. Distributed Work. MIT Press, Cambridge, MA, 311-333.

Williams, K. Y., C. A. O’Reilly. 1998. Demography and diversity in organizations: A review of 40 years of research. B. M. Staw, L. L. Cummings, eds. Research in Organizational Behavior, Vol. 20. JAI Press, Greenwich, CT, 77-140.

Xie, J., X. M. Song, A. Stringfellow. 1998. Interfunctional conflict, conflict resolution styles, and new product success: A fourculture perspective. Management Sci. 44 192-206.

Zajonc, R. B. 1968. Attitudinal effect of mere exposure. J. Personality Soc. Psych. 9 2-17. 